\title{
Placing LDACS-Based Ranging Sources for Robust RNP 1.0 Accuracy En-Route
}

\author{
Giuseppe Battista, Rachit Kumar, Elisabeth Nossek and Okuary Osechas \\ German Aerospace Center (DLR), Institute of Communications and Navigation, \\ Oberpfaffenhofen, Germany, Email: Giuseppe.Battista@dlr.de
}

\begin{abstract}
Global Navigation Satellite Systems (GNSS) are becoming the primary means of navigation for civil aviation. Nevertheless, concerns about GNSS outages remain, driving the need for Alternative Positioning, Navigation and Timing (APNT) systems to provide availability and continuity for performancebased navigation services. Although the existing Distance Measuring Equipment (DME) infrastructure is able to provide Required Navigation Performance (RNP) 1.0 accuracy, it is not robust for individual station outages. Additionally, we show that in the European airspace DME is reaching the capacity limit. To address these two problems, we propose a methodology based on modular APNT. In the presented approach, the complementary ranging sources are optimally placed to obtain robustness. It is assumed that the L-Band Digital Aeronautical Communications System (LDACS) can provide this capability. As shown in the results, the modular APNT system is able to provide robust RNP 1.0 coverage for Germany using 17 new LDACS ground stations to complement the network of 73 existing DME installations in Germany.

Index Terms-APNT, LDACS, RNP, DME
\end{abstract}

\section{INTRODUCTION}

Current Air-Traffic Management (ATM) systems increasingly rely on Global Navigation Satellite Systems (GNSS) as a primary means of navigation. The adoption of GNSS has enabled a new class of service: Required Navigation Performance (RNP). RNP provides improved safety and efficiency, compared to conventional navigation based on terrestrial navigation aids (navaids). Several RNP service levels exist and they are identified with a number that indicates the $95 \%$ error, in nautical miles, that the system can tolerate; for example RNP 1.0 indicates that the total system error will be within 1 NMi of the indicated position $95 \%$ of the time.

While the existing navigation infrastructure is able to meet current demand levels, it leaves little room for disruptions. GNSS-based services are known to be vulnerable to disruptions, from jamming or spoofing, while conventional navaids do not provide the same level of performance. Navaid-based services are not as accurate as GNSS-based services are and, compared with GNSS, they have reduced capacity for handling aircraft.

\section{A. The Challenge}

A likely short-term solution to providing APNT-based RNP is to rely on Distance Measuring Equipment (DME). There are two key limitations that a future APNT system will need to address, if it is to provide service continuity during GNSS outages:

- lack of robustness in providing RNP, and

- limited capacity in handling traffic.

The lack of robustness is best illustrated in Figure 1. The claim that the DME infrastructure can provide RNP 1.0, as modeled in [1] and [2], assumes that all measurements are available and fault-free at any given time. In order to account for an ability to cope with dropped or faulted measurements we propose analyzing the performance of the DME network in a leave-one-out fashion. This technique has been applied in GNSS applications related to Receiver Autonomous Integrity Monitoring (RAIM) [3]. We show the resulting leave-oneout positioning error for the current DME infrastructure over Germany in Figure 1, while Figure 2 gives a histogram of the same error, as a way of highlighting the fact that at most $20 \%$ of the airspace is able to support RNP 1.0.

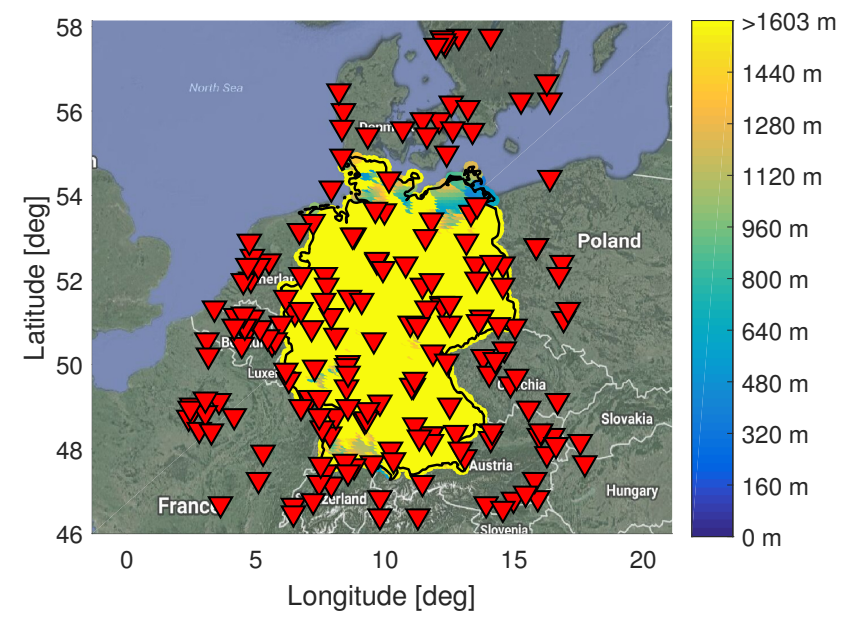

Fig. 1. Leave-One-Out 2- $\sigma$ of NSE in Germany for DME-standalone network at FL100

Figure 3 highlights the capacity limitation imposed by the DME network. The map shows the number of aircraft a DME site has in view at a peak time on 30 July 2015. Some stations have more than 100 aircraft in view. Conventional DME ground infrastructure can support somewhere between 100 and 200 aircraft, depending on several different parameters. This map shows that in parts of Germany the DME network is 

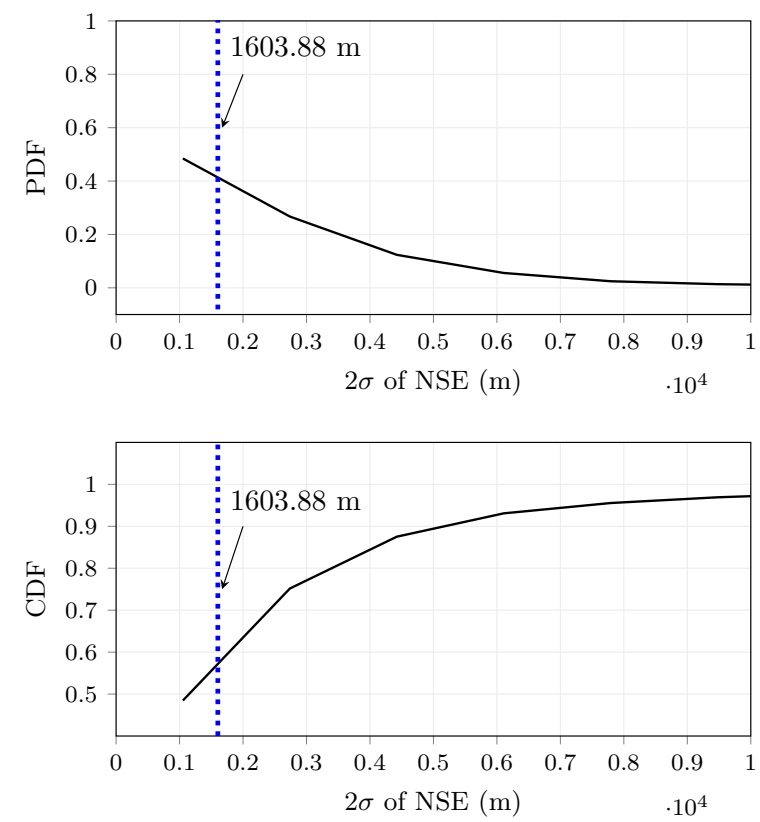

Fig. 2. Leave-One-Out 2- $\sigma$ of NSE in Germany for DME-standalone network at FL100

starting to become a factor in limiting air traffic throughput. This observation is particularly worrisome in the light of forecasts that the demand for air traffic will continue to increase in the near future [4].

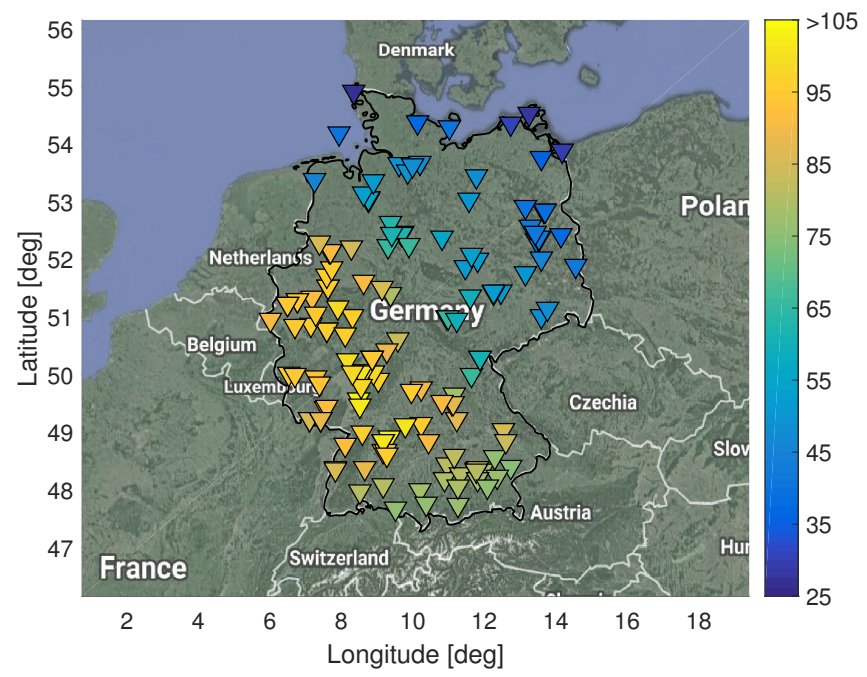

Fig. 3. Traffic load map of DME network in a busiest hour in a day (DFS survellaince data - 2015).

\section{B. Modular APNT}

The aviation community has identified a need for terrestrially-based navigation services that support RNP. While several candidates have been discussed in the literature [5], no single technology (or set of technologies) has established itself enough to warrant significant implementation efforts. On the contrary, it is conceivable that APNT systems would not require specific technologies, but instead accommodate many different technologies are hybridized into one modular position solution [6]. One particular instance of hybrid modular APNT that has received attention in the past is the hybridization of DME with the L-Band Digital Aeronautical Communications System (LDACS) [2]. The LDACS signal has been demonstrated to support terrestrial ranging with an accuracy greater than that of DME [7] and can be conservatively modeled with a Gaussian distribution of standard deviation $20 \mathrm{~m}(2 \sigma)$ [7].

A hybrid solution, based on the combination of DME with LDACS, offers a compromise. On the one hand, the DME-only solution is shown to not be robust; on the other hand, an LDACS-only navigation system will not be available in the short term. The hybrid-modular approach potentially reconciles the need for a robust navigation system with a significantly shorter timeline than an LDACS-based navigation system.

One of the questions this paper answers is how many LDACS stations are needed to complement the existing DME network of stations. To answer the question we develop a methodology for placing new LDACS stations. The optimization minimizes the number of new stations, while providing robust RNP coverage over $95 \%$ of the German airspace at an altitude of $10.000 \mathrm{ft}$.

\section{APNT BACKGROUND}

In this chapter we introduce the key concepts behind the proposed methodology. The chapter starts with a short introduction of RNP operations. Later, a description of the NSE computation model is given. Finally, we briefly introduce two technologies that would potentially enable modular APNT: DME and LDACS.

\section{A. Required Navigation Peformance (RNP)}

Area Navigation (RNAV) is a method for navigation based on instrument flight rules (IFR) which permits aircraft operation on any desired path, provided that the selected path is within the coverage of NAVAIDS. RNAV is enabled by defining a set of way points between source and the destination. The position is estimated using the on-board computer. The tolerance in error of the position estimate is different for different segments of flight and is mentioned in the RNAV specification. The specification of RNAV 1 means that the total system error should not exceed $1 \mathrm{NM}(2 \sigma)$. The PBN concept represents a shift from sensor-based to performance-based navigation. With the advent of GNSS, a new concept of RNP was introduced. On top of maintaining the total system error requirement of RNAV, RNP also requires on-board monitoring and alerting capability [8]. The adoption of RNP improves safety and efficiency.

1) Total System Error (TSE): is the difference between the true position and the desired position, as shown in fig. 4 . According the (1), TSE is computed as squared sum of: Path Definition Error (PDE), the Flight Technical Error (FTE) and the Navigation System Error (NSE). 


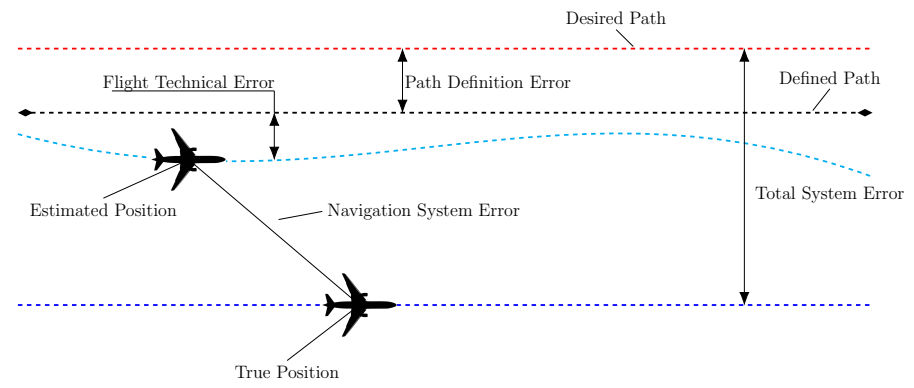

Fig. 4. Total System Error.

$$
T S E=\sqrt{F T E^{2}+N S E^{2}+P D E^{2}}
$$

It is important to note, that both RNP and RNAV requirements are only for TSE.

The Flight Management System (FMS) has to perform integrity checks during way-points definition, this makes PDE negligible. FTE is related to pilot or autopilot ability to follow the defined path or track, including any displayed error (e.g. course deviation indicator error). The FTE requirements for different phases of flight are reported in tab. I [9].

TABLE I

FTE SPECIFICATIONS

\begin{tabular}{l|l|l|l}
\hline Flight Phases & Manual (NM) & $\begin{array}{l}\text { Flight Direc- } \\
\text { tor (NM) }\end{array}$ & $\begin{array}{l}\text { Autopilot } \\
\text { (NM) }\end{array}$ \\
\hline Oceanic & 2.0 & 0.5 & 0.25 \\
En Route & 1.0 & 0.5 & 0.25 \\
Terminal & 1.0 & 0.5 & 0.25 \\
Approach & 0.5 & 0.25 & 0.125 \\
\hline
\end{tabular}

Assuming a FTE of 0.5 NM:

- To support RNP 1.0 the NSE is required to be less than $1603 \mathrm{~m}(2 \sigma)$;

- to support RNP 0.3 the NSE is required to be less than $307 \mathrm{~m}(2 \sigma)$.

\section{B. Modular APNT}

1) NSE Model: following notations are used:

- $\mathbf{x}$ is the user position;

- $b$ is the clock bias;

- $\mathbf{s}_{i}$ is the position of $i$-th station;

- $\rho_{i}$ is the two-way range measurement for $i$-th ranging source;

- $k$ is the total number of two-way ranging sources in view;

- $N-k$ is the number of one-way ranging sources in view;

- $\rho_{k}$ is the one-way pseudorange measurement for $k$-th ranging source;

- $N$ is the total number of stations in view.

Fig. 5 shows the setup for APNT. From the pseudo-range equations, the residual function can be defined as:

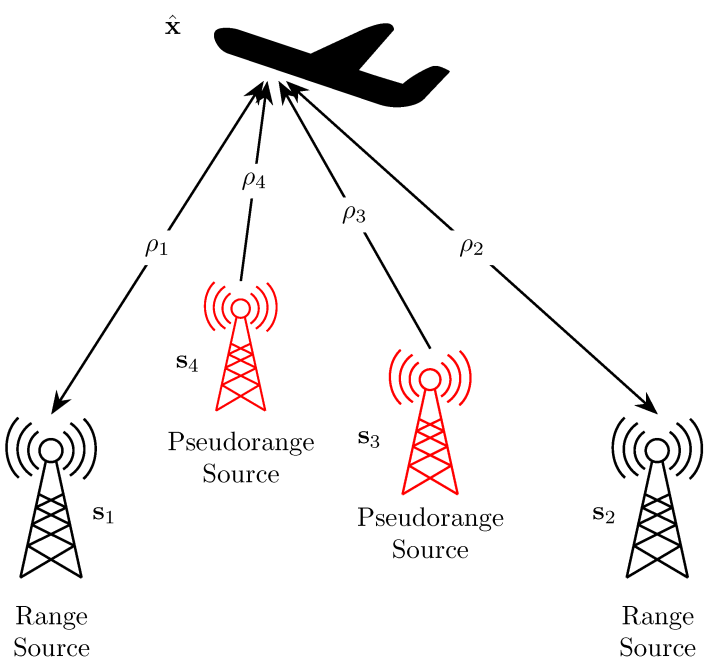

Fig. 5. Positioning with pseudoranging and ranging measurements.

$$
\mathbf{F}(\mathbf{x}, b)=\left(\begin{array}{c}
\rho_{1}-\left\|\mathbf{s}_{1}-\mathbf{x}\right\| \\
\vdots \\
\rho_{k}-\left\|\mathbf{s}_{k}-\mathbf{x}\right\| \\
\rho_{k+1}-\left(\left\|\mathbf{s}_{k+1}-\mathbf{x}\right\|+b\right) \\
\vdots \\
\rho_{N}-\left(\left\|\mathbf{s}_{N}-\mathbf{x}\right\|+b\right)
\end{array}\right)
$$

The position solution is given by the vector $\hat{\mathbf{x}}$ (estimated position) and the value $\hat{b}$ (estimated clock bias) which minimizes the norm of residual function (2) [10]. As the function is non-linear, it is made linear using Taylor series, which results in geometry matrix $\mathbf{G}(\mathbf{x})$ shown in equation (3). The geometry matrix is the Jacobian matrix of $\mathbf{F}$ :

$$
\mathbf{G}(\mathbf{x})=\frac{d \mathbf{F}(\mathbf{x}, b)}{d(\mathbf{x}, b)}=\left(\begin{array}{cc}
\frac{1}{\left\|\mathbf{s}_{1}-\mathbf{x}\right\|}\left(\mathbf{s}_{1}-\mathbf{x}\right)^{T} & 0 \\
\vdots & \\
\frac{1}{\left\|\mathbf{s}_{k}-\mathbf{x}\right\|}\left(\mathbf{s}_{k}-\mathbf{x}\right)^{T} & 0 \\
\frac{1}{\left\|\mathbf{s}_{k+1}-\mathbf{x}\right\|}\left(\mathbf{s}_{k+1}-\mathbf{x}\right)^{T} & -1 \\
\vdots & \\
\frac{1}{\left\|\mathbf{s}_{N}-\mathbf{x}\right\|}\left(\mathbf{s}_{N}-\mathbf{x}\right)^{T} & -1
\end{array}\right)
$$

Using a starting vector $\left(\mathbf{x}_{0}, b_{0}\right)^{T}$ which is sufficiently close to the true user position, the position solution is obtained iteratively by:

$$
\begin{aligned}
\left(\begin{array}{c}
\mathbf{x}_{n+1} \\
b_{n+1}
\end{array}\right)= & \left(\begin{array}{l}
\mathbf{x}_{n} \\
b_{n}
\end{array}\right)- \\
& \left(\mathbf{G}\left(\mathbf{x}_{n}\right)^{T} \mathbf{W G}\left(\mathbf{x}_{n}\right)\right)^{-1} \mathbf{G}\left(\mathbf{x}_{n}\right)^{T} \mathbf{W F}\left(\mathbf{x}_{n}, b_{n}\right)
\end{aligned}
$$

Where $\mathbf{W}$ is the weighting matrix: 


$$
\mathbf{W}=\left(\begin{array}{ccccc}
\frac{1}{\sigma_{\rho_{1}}^{2}} & & \cdots & & 0 \\
\vdots & \ddots & & & \vdots \\
0 & & \frac{1}{\sigma_{\rho_{k}}^{2}} & & 0 \\
\vdots & & & \ddots & \vdots \\
0 & & \cdots & & \frac{1}{\sigma_{\rho_{N}}^{2}}
\end{array}\right)
$$

The weighting matrix diagonal elements are inverse of range error variance. The weighting matrix is also used in GPS based positioning, as low elevation satellites have large error and hence have low weight in position solution. In our assessment we use the $\mathbf{W}$ matrix for the hybrid configuration, where position solution is obtained combining pseudorange and range measurements. The diagonal elements of the weighting matrix in this case depends on the range error uncertainty of the ranging sources.

As mentioned in [3], we can define $\Delta \mathbf{x}=\hat{\mathbf{x}}-\mathbf{x}$ and $\Delta b=$ $\hat{b}-b$, and compute the covariance matrix $\mathbf{H}$ as:

$$
\begin{aligned}
\operatorname{cov}\left[\begin{array}{c}
\Delta \mathbf{x} \\
\Delta b
\end{array}\right] & =\operatorname{cov}\left[\begin{array}{c}
\hat{\mathbf{x}} \\
\hat{b}
\end{array}\right]=\left(\mathbf{G}^{T} \mathbf{W G}\right)^{-1}=\mathbf{H} \\
\mathbf{H} & =\left[\begin{array}{cccc}
\sigma_{E}^{2} & \cdot & \cdot & \cdot \\
\cdot & \sigma_{N}^{2} & \cdot & \cdot \\
\cdot & \cdot & \sigma_{D}^{2} & \cdot \\
\cdot & \cdot & \cdot & \sigma_{T}^{2}
\end{array}\right]
\end{aligned}
$$

Given the matrix $\mathbf{H}$ in local frame, the uncertainity $(\sigma)$ of navigation system error (NSE) can be computed by equation (9):

$$
2 \sigma_{N S E}=\sqrt{H_{11}+H_{22}}=\sqrt{\sigma_{E}^{2}+\sigma_{N}^{2}}
$$

2) Distance Measuring Equipment (DME): is a two-way ranging system operating in the L-band of radio frequencies between 960-1215 MHz. The DME operating principle is simple. The slant range is computed in the aircraft by calculating the round-trip time between the interrogations from an on-board transmitter and replies to those interrogations from a ground station [11]. Both, transmitted interrogation and received replies, are pulse pairs with a separation in frequency of $63 \mathrm{MHz}$.
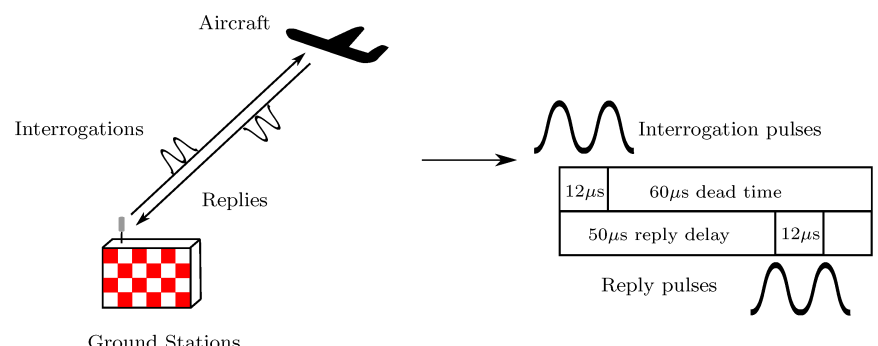

Fig. 6. DME Transponder Operation

The round trip delay is given as difference of known interrogation time, and reception time, obtained by correlation between pulse pairs. According to FAA Advisory Circular [12] the standard deviation of the ranging measurement error $\sigma_{R}$ is defined by equation (10).

$$
\sigma_{R}^{2}=\sigma_{\text {sis }}^{2}+\sigma_{\text {air }}^{2}
$$

where $\sigma_{\text {sis }}$ is signal-in-space error (distance independent) of $0.05 \mathrm{NM}, \sigma_{\text {air }}$ is the error due to atmospheric delays equal to $\max \left\{0.085,0.00125 \cdot D_{i}\right\}$. From (10), we get $2 \sigma_{R}=182 \mathrm{~m}$ at $68 \mathrm{NM}$.

3) L-Band Digital Aeronautical Communication System $(L D A C S)$ : is a candidate for future aeronautical communications. The ranging capability of LDACS allows to enable navigation services for ATM. LDACS uses Orthogonal FrequencyDivision Multiplexing (OFDM) for modulation and operates in the aeronautical L-band (960-1164 MHz). Each station is assigned a bandwidth of $500 \mathrm{kHz}$. LDACS deployment in L-band can be done in two different ways: inlay scenario and non-inlay scenario. From the point of view of spectrum availability the most preferable approach is the inlay scenario, as in fig 7, where LDACS channels are placed between the existing DME channel grid of $1 \mathrm{MHz}$ with an offset of $500 \mathrm{kHz}$ with respect to DME center frequencies. This option is convenient because it is not necessary to change the existing DME frequency allocation. LDACS has a ranging uncertainty of $20 \mathrm{~m}(2 \sigma)$ [13]. Thiasiriphet et al. has shown that with Doppler smoothing (low complexity approach) ranging performance can be improved: $\sigma_{R}$ 3-6 $\mathrm{m}(2 \sigma)$ [7].

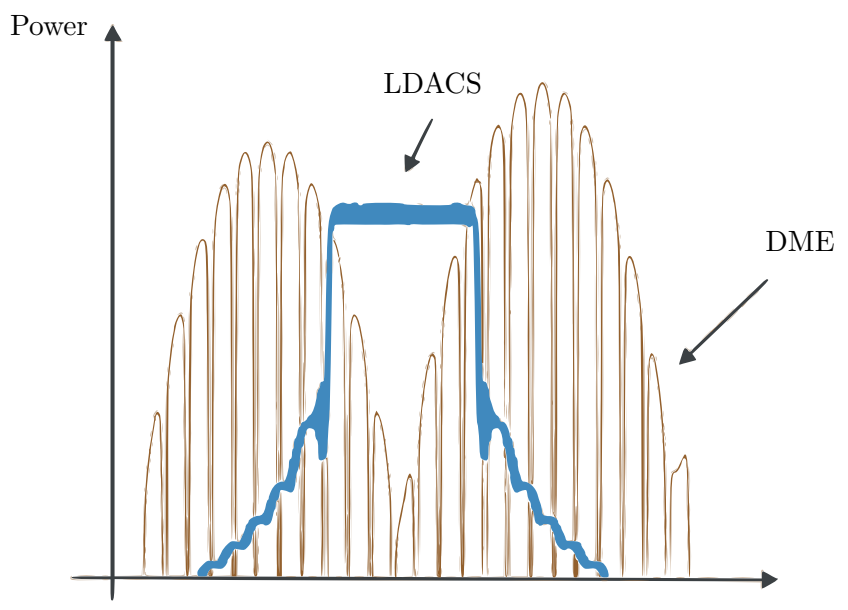

Frequency

Fig. 7. In-lay option for spectral deployment of LDACS.

\section{Placement Strategy}

We propose a new method to select optimal sites from VHF-comm network to place LDACS, in order to have a hybrid DME-LDACS network to provide a robust RNP 1.0 accuracy over German airspace. The selection of sites, in computational complexity theory terms, is called nondetermistic polynomial problem [14]. For this kind of problem, no algorithms are known to provide an optimal solution. The 
only approach guaranteed to find the global optimum is to use an exhaustive search, which is computationally intensive. The method presented here is more computationally efficient than the exhaustive search. In this configuration, we combine the pseudo-range measurement from LDACS and two-way range measurements from DME.

The notion that LDACS ground stations would be implemented at currently operational VHF-comm sites presents several advantages. For one, LDACS is a communications system and it would present similar siting constraints to existing communications systems. These constraints might include availability of power and high-speed internet, good visibility of the sky, ease of access for maintenance purposes, etc. For another, the density of the network of VHF-comm sites is far greater than that of most other aeronautical CNS system. The dense network provides a high degree of flexibility when selecting potential sites for LDACS ground stations, which translates into a more uniform distribution of the provided NSE than a less dense set of ground stations would. The following section describes the algorithm for selecting the subset of VHF-comm stations to be upgraded to LDACS ground stations and provide robust RNP coverage.

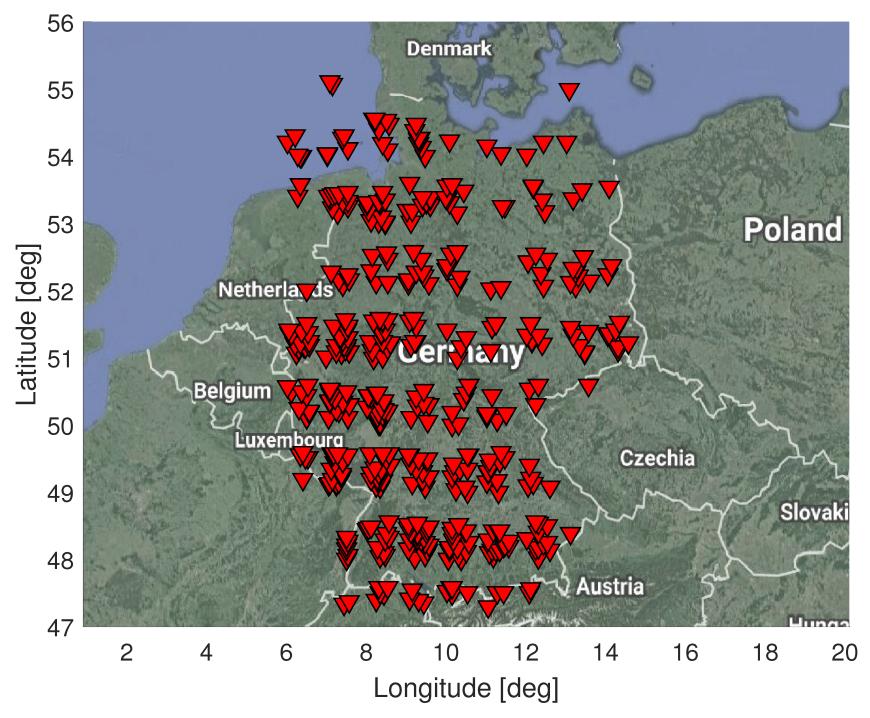

Fig. 8. VHF-comm stations in Germany.

\section{A. Proposed Algorithm}

The NSE model depends on uncertainties along the North and East direction, which in turn will depend on ranging errors and relative geometry between ground stations and aircraft. In fig. 9(a), a poor geometry is shown. The poor geometry is due to the location of the ground stations. The uncertainty in user position is wide (dashed area in figure). The position uncertainty can be reduced when the ground stations are orthogonal to each other, as shown in fig. 9(b) where the error area is smaller compared to the poor geometry configuration. The best possible transmitters geometry in terms of NSE is defined by all ground stations spaced equally in azimuth direction, as shown in fig. 10. It is important to underline here, that when the stations are at the horizon, experience has shown that the signals are affected by higher tropospheric delays and multipath effects. Hence, in order to bound the NSE, it will be more important have a good geometry for stations far from user since the closer stations have less contributions on position error. [3].

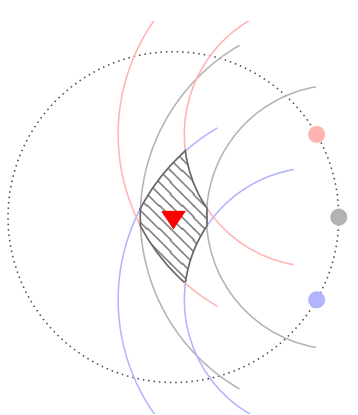

(a) Poor geometry

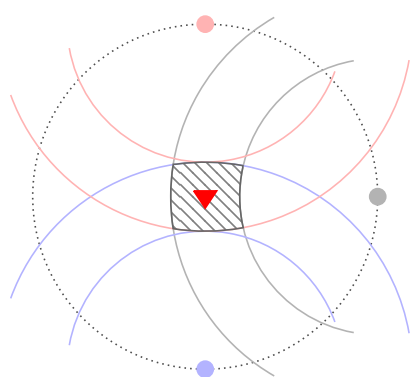

(b) Good geometry
Fig. 9. Influence of relative geometry receiver-transmitter on position uncertainty.

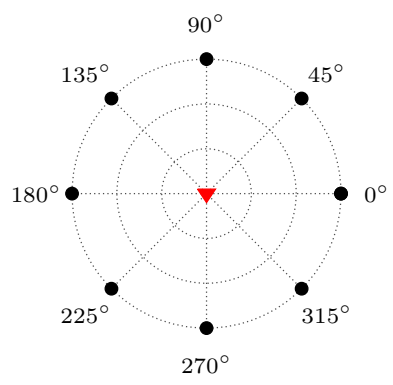

(a) 8 Ground Stations

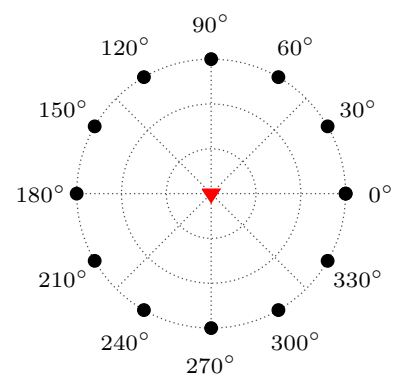

(b) 12 Ground Stations
Fig. 10. Optimal geometry for ground stations with respect the aircraft (center).

The main goal of the algorithm is to select good subset of VHF-comm sites to place LDACS in order to achieve an NSE low enough to support the desired RNP type, with a small number of stations. The algorithm, fig. 11, consists of the following steps:

1) pick a point $j$ on the grid as a virtual aircraft;

2) select DME and already LDACS placed stations in view;

3) check if NSE is lower than the required threshold;

4) divide the ENU plane into sectors, as in Fig. 11;

5) compute for each region the ratio between the number of stations in the area and total number of stations in view;

6) find VHF-comm sites in the outer regions with lower ratio, with a cardinality of 2 ;

7) compute NSE of new hybrid configuration;

8) check if NSE is lower than the required threshold;

9) if the answer is no: increase cardinality, and go again to step 5. If the answer is yes stop the search and continue with next point on the grid.

Fig. 12 shows a block diagram of the proposed algorithm. 


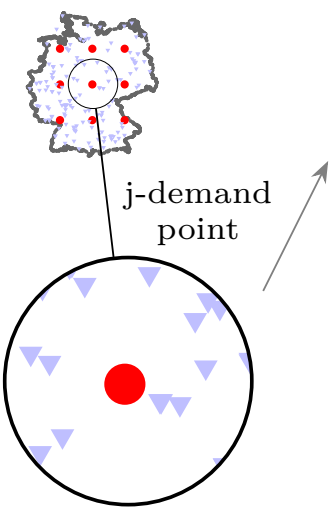

DME stations

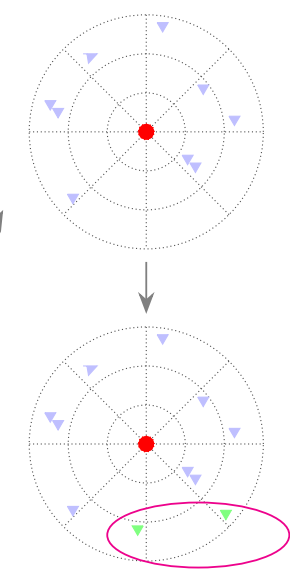

New LDACS stations

Fig. 11. Selection of optimal LDACS sites.

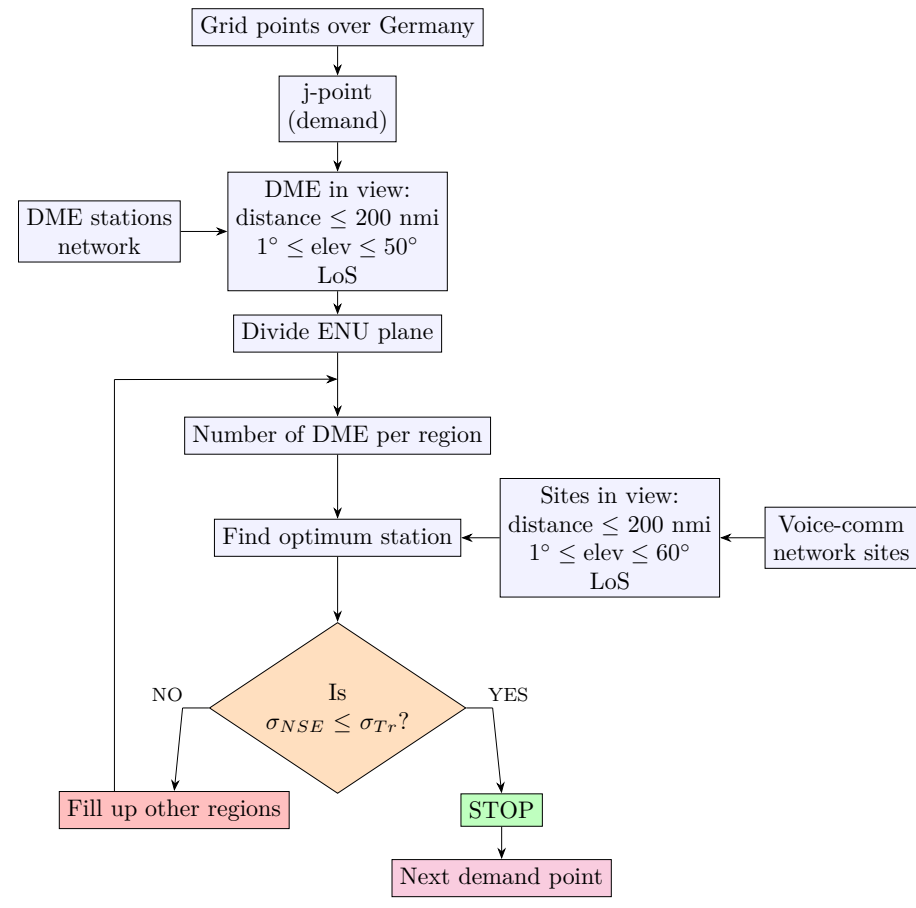

Fig. 12. Flowchart of selection algorithm.

\section{IMPLEMENTATION AND RESUltS}

In this chapter we define the various parameters and constraints used in the optimization algorithm, followed by the results of the algorithm implementation in two scenarios.

\section{A. Algorithm Input Parameters}

The following assumptions are used in algorithm:

- DME specifications:

- 200 NM maximum coverage distance;

- $1 \mathrm{deg}$ minimum elevation, $40 \mathrm{deg}$ maximum elevation;

- $2 \sigma_{R}$ range uncertainty given by eq. (10);
- 200 aircraft handling capacity.

- LDACS specifications:

- 160 NM maximum coverage distance;

- $0.5 \mathrm{deg}$ minimum elevation, $60 \mathrm{deg}$ maximum elevation;

- $2 \sigma_{R}$ range uncertainty equal to $20 \mathrm{~m}$;

- Unlimited aircraft handling capacity (pseudo-ranging system).

- 3100 m flight altitude, i.e. FL100.

- $\approx 300$ DME sites in Germany and neighboring countries, $\approx 580$ VHF-comm sites.

- Visibility analysis (line-of-sight between ground station and aircraft) using Digital Elevation Models (DEM) taken from U.S. National Aeronautics and Space Administration (NASA) and Japanese Ministry of Economy (METI). The DEM is ASTER Global Digital Elevation Model with vertical RMS between $10 \mathrm{~m}$ and $25 \mathrm{~m}$ [15].

Maximum coverage distance, minimum and maximum elevation, line-of-sight are shown in fig. 13.

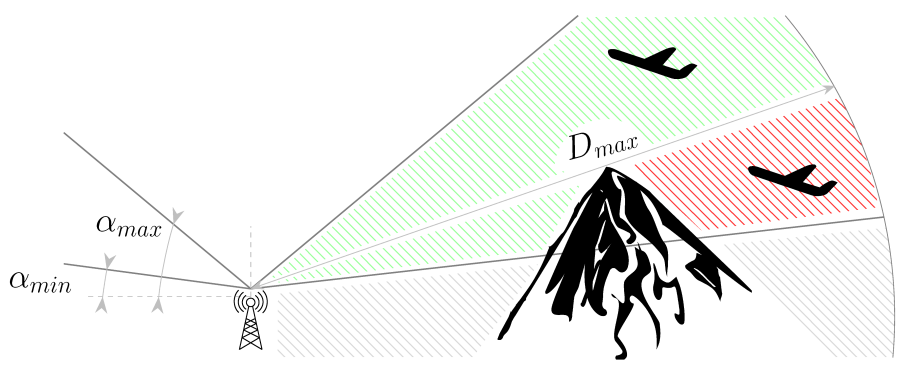

Fig. 13. Representation of maximum coverage distance $\left(D_{\max }\right)$, elevation mask $\left(\alpha_{\min }-\alpha_{\max }\right)$ and line-of-sight between ground station and aircraft.

\section{B. Algorithm Results}

The algorithm is tested on two different spaced grids, with leave-one-out fashion:

- First scenario: $2.294 \mathrm{deg}$ along longitude and $1.945 \mathrm{deg}$ along latitude. Grid of $5 \times 5$ points over Germany. Results are shown in fig. 14 and 15;

- Second scenario: 1.019 deg along longitude and $0.865 \mathrm{deg}$ along latitude. Grid of $10 \times 10$ points over Germany. Results are shown in fig. 16 and 17.

Using the algorithm described earlier, the number of LDACS stations placed in first scenario is 17 , and in second scenario is 84 . Fig. 15 shows that with only 17 stations placed in Germany we achieve a NSE of $400 \mathrm{~m}$ for more than $80 \%$ of airspace at FL 100. With second configuration, where we place 84 stations, we achieve an error less than $307.1 \mathrm{~m}$ for more than $95 \%$ of the area as shown in 17 .

\section{Discussion}

The notion of resilience, inherent to the methodology of this paper, breaks with the traditional view that a terrestrial navigation aid is unquestionably reliable. The way in which conventional navaids are used assumes that their readings are completely trustworthy. That is an assumption that works 


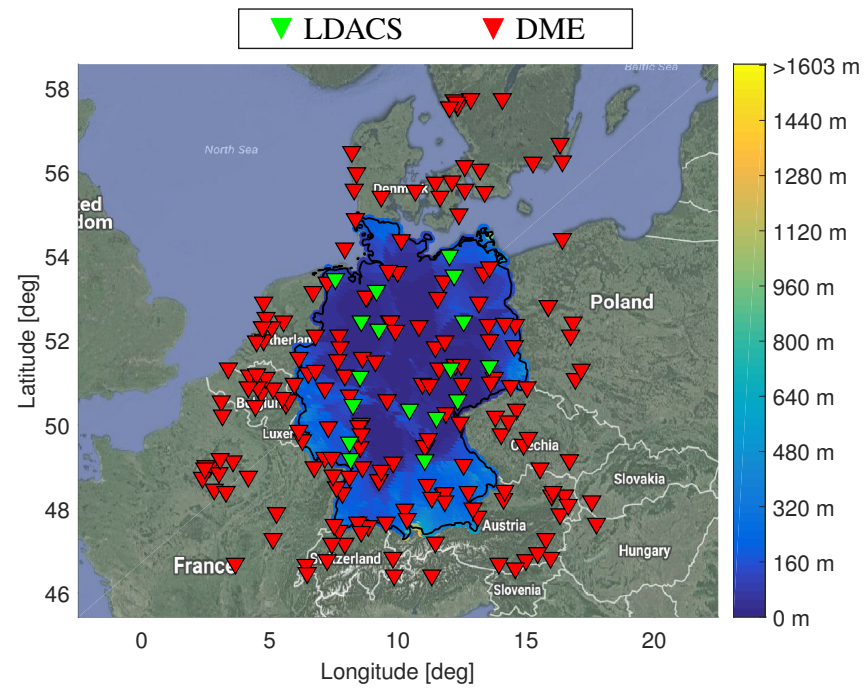

Fig. 14. Leave-on-out $2 \sigma$ of NSE (in meter) placing LDACS with heuristic method, using a grid of $5 \times 5$ points, at FL100.
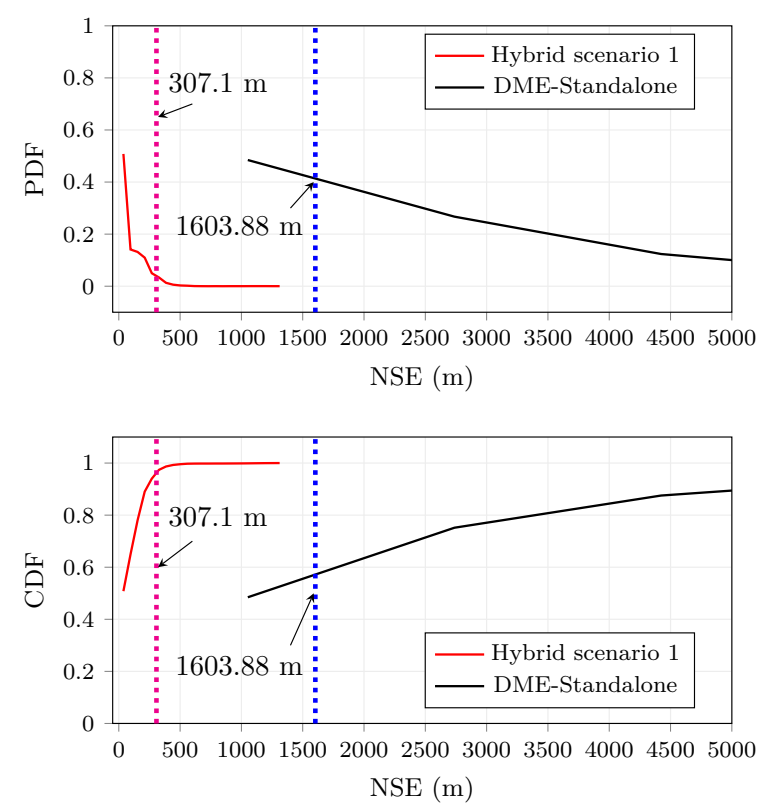

Fig. 15. Leave-on-out $2 \sigma$ of NSE distribution (in meter) placing LDACS with heuristic method, using a grid of $5 \times 5$ points, at FL100.

well with a human pilot in the loop, but it does not support the kind of automated decision-making envisioned for future operations, whether for unmanned systems or for manned systems under IFR.

In this context, one important detail to keep in mind about the results of Figures 14-17 is that they include a measure of redundancy. The positioning performance is computed in a leave-one-out fashion, as a way of accounting for singlesource outages in the position solution. It is debatable, whether leave-one-out is the most appropriate criterion to account for imperfect distance measurements. However, the alternative of assuming that the signals from all ground stations in view

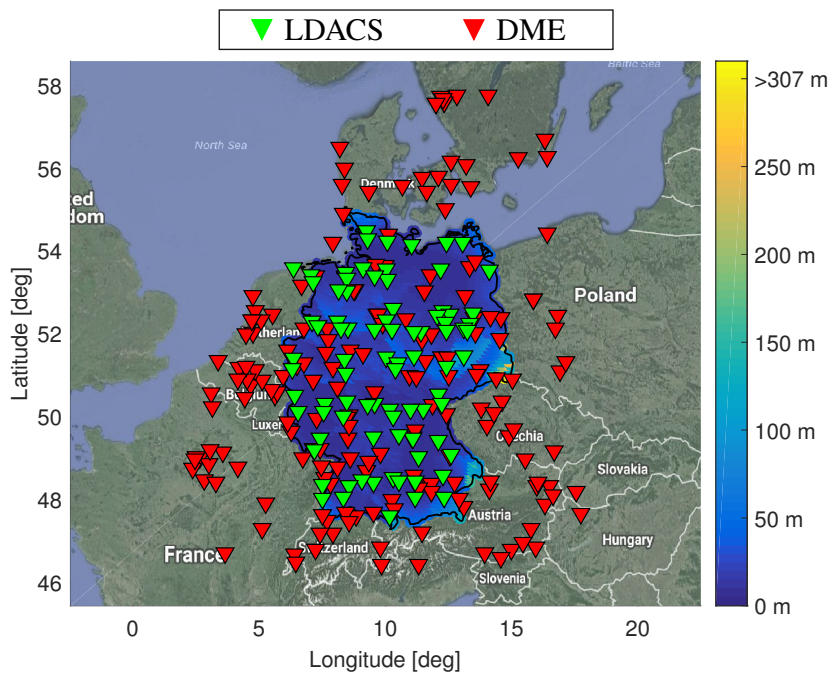

Fig. 16. Leave-on-out $2 \sigma$ of NSE (in meter) placing LDACS with heuristic method, using a grid of $10 \times 10$ points, at FL100.
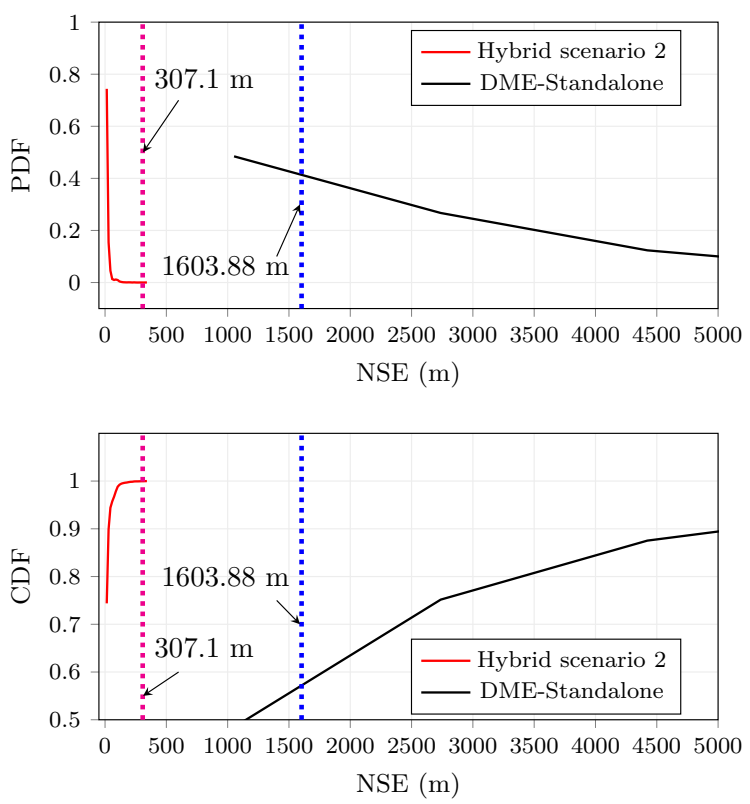

Fig. 17. Leave-on-out $2 \sigma$ of NSE distribution (in meter) placing LDACS with heuristic method, using a grid of $10 \times 10$ points, at FL100.

are received perfectly is also unrealistic, as the integrity of terrestrial ranging is not a settled matter. Between these two options, the former one is more conservative and, therefore, we propose that as a more desirable route towards APNT integrity.

A central result of this paper is the number of LDACS stations needed to complement DME in providing RNP accuracy over Germany. There are two values, each associated with a different RNP type: 17 LDACS stations will enable robust RNP 1 over Germany. For RNP 0.3 the number of LDACS stations grows to 89 . When put in the context of providing data links, which is the original mission of LDACS, it is worth noting that the current network of VHF Data Link 
Mode 2 (VDL2) uses 18 ground stations to cover Germany [16]. While the band width and data rates provided by VDL2 are not necessarily comparable to those of LDACS, there is a striking similarity between these two numbers. Arguably, providing a new aeronautical communication service is easier, if the available infrastructure already supports aeronautical communications. The question whether the sites of VDL2 stations provide adequate spatial diversity to support a hybrid APNT solution remains a topic for future work.

Another benefit of our method is its flexibility, as it can easily be adapted to address service levels other than RNP 1 or RNP 0.3 . The algorithm in Figure 12 simply needs to use a different $\sigma_{T r}$, which could even be made to vary with geographical location, allowing for different performance levels.

\section{SUMMARY}

The placement of LDACS using the algorithm increases the number of stations in view for each point in the considered airspace volume. This increases the availability of alternative means of navigation during a GNSS outage. The proposed hybrid LDACS-DME system brings high accuracy over Germany, as shown in figures 15 and 17 . In the first simulation, with only 17 LDACS stations, the area in which the NSE is greater than $200 \mathrm{~m}$ is reduced by half, in the second configuration, with 84 LDACS stations placed. LDACS has two additional advantages:

1) it is one-way ranging system, which means unlimited aircraft handling capacity;

2) it adds redundancy to the APNT service, making it more robust.

The main concern about assuring integrity for ground based ranging system is the detection of multipath propagation, which remains a topic for future work. Nevertheless, to provide RNP 1.0 service, integrity assessments needs to be done for hybrid LDACS-DME system and the proposed leave-one-out criterion is a first step towards a more robust APNT. Moreover, there is a need to implement a fault monitor on-board and/or on-ground.

\section{ACKNOWLEDGMENT}

The authors would like to thank Boubeker Belabbas (DLR) for his valuable inputs. The authors would also like to thank Thanawat Thiasiriphet (DLR) for providing LDACS related data and DFS for providing the surveillance data.

\section{REFERENCES}

[1] V. V. S. Berz, G., "Can Current DME Support PBN Operations with Integrity?" presented at the Proceedings of the 26th International Technical Meeting of The Satellite Division of the Institute of Navigation, 2013, pp. 233-250.

[2] R. Kumar, G. Battista, and O. Osechas, "Demand-based Placement of LDACS Ground Stations to Achieve RNP 0.3 Accuracy for APNT," pp. 1227-1241, 2017.

[3] P. Misra and P. Enge, Eds., Global Positioning System: Signals, Measurements, and Performance. Ganga-Jamuna Press, 2012, vol. 2nd edition.
[4] EUROCONTROL, "Long-Term Forecast - Flight Movements 20102030," EUROCONTROL, 96 Rue de la Fusee, B-1130 Brussels, Tech. Rep., 2010.

[5] S. Lo, "Pseudolite Alternatives for Alternate Positioning, Navigation, and Timing (APNT)," FAA White Paper, August, 2012.

[6] O. Osechas, E. Nossek, B. Belabbas, and M. Meurer, "A Modular Approach to Integrity for APNT," in 29th International Technical Meeting of The Satellite Division of the Institute of Navigation (ION GNSS+ 2016), 2016

[7] T. Thiasiriphet, N. Schneckenburger, and M. Schnell, "Ranging with LDACS: Results from Measurement Campaign," in 2016 Integrated Communications Navigation and Surveillance (ICNS), 2016, pp. 1-18.

[8] ICAO, "Performance-based Navigation (PBN) Manual," ICAO, 999 University Street, Montreal, Quebec, Canada, Tech. Rep., 2008.

[9] RTCA, "DO-208: Minimum Operational Performance Standards For Airborne Supplemental Navigation Equipment Using Global Positioning System," RTCA Inc., Washington DC, Tech. Rep., 1996.

[10] E. Nossek, O. Osechas, and M. Meurer, "Error Analysis for the Combination of Angular, Ranging, and Barometric Measurements," in ION GNSS+ 2016, 2016.

[11] D. R. C. Robert J. Kelly, Ed., Distance Mesuring Equipment and its Evolving Role in Aviation, ser. Advances in Electronics and Electron Physics. Academic Press, 1986, vol. 68.

[12] FAA, "Advisory Circular - U.S. Terminal and En Route Area Navigation (RNAV) Operations," FAA, Tech. Rep., 2007.

[13] M. Schnell, U. Epple, D. Shutin, and N. Schneckenburger, "LDACS: Future Aeronautical Communications for Air-Traffic Management," IEEE Communications Magazine, vol. 52, no. 5, pp. 104-110, 2014.

[14] E. Kim, "Investigation of APNT Optimized DME/DME Network Using Current State-Of-the-Art DMEs: Ground Station Network, Accuracy, and Capacity," in Proceedings of the 2012 IEEE/ION Position, Location and Navigation Symposium, 2012, pp. 146-157.

[15] NASA. (2017, Jan.) ASTER NASA Digital Elevation Model. [Online]. Available: https://lpdaac.usgs.gov/

[16] "Air Navigation Plan - European Region. Volume II, Facilities and Services Implementation Document (FASID). Part IV - CNS. Supplement Table COM-2." ICAO, Tech. Rep., 2007.

\section{BIOGRAPHIES}

Giuseppe Battista received his Bachelor and Master degree in Aerospace Engineering from University of Naples Federico II. He joined navigation Integrity Group in the Institute of Communications and Navigation at the German Aerospace Center (DLR) in April 2016 and is involved in the research on APNT systems.

Rachit Kumar is a researcher in Department of Navigation at German Aerospace Center (DLR). His research work is mainly focused on APNT techniques. He finished his MSc in Space Science and Technology from Aalto University, Finland. Prior to this he did his first MSc in Control System Engineering from The University of Sheffield, United Kingdom.

Dr. Elisabeth Nossek is a member of the Navigation Integrity Group of the Institute of Communications and Navigation at the German Aerospace Center (DLR) in Oberpfaffenhofen. Her current research interests focus on APNT. She received the diploma and Ph.D. degrees in Mathematics at RWTH Aachen University.

Dr. Okuary Osechas is a researcher with the Institute of Communications and Navigation at DLR. He received a Diploma in Electrical Engineering from Karlsruhe University and a Ph. D. in Electrical Engineering from Tufts University. 
His research interests focus on navigation integrity and security, both in satellite navigation and in alternative systems. 